\title{
Le résumé structuré : un outil de lecture, d'évaluation et de rédaction
}

\author{
Georges BORDAGE*, Serge QUERIN* *
}

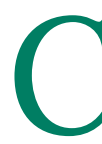

\section{7}

la fin des années soixante que les rédacteurs en chef de revues scientifiques ont commencé à joindre des résumés à leurs articles. Vingt ans plus tard, un groupe de travail a plaidé en faveur de résumés plus informatifs basés sur des critères reconnus de lecture critique de la littérature médical $\mathrm{e}^{\mathrm{l}}$.

Le groupe de travail a proposé un résumé en sept points composé de 250 mots. Bientôt, un huitième élément a été ajoutén ${ }^{2,3}$ :

1 - But : la question de recherche.

2 - Plan de recherche (type d'étude).

3 - Contexte : Ie milieu et le niveau de soins (ou d'enseignement).

4 - Participants : Ia méthode de sélection et le nombre de participants qui ont commencé et qui ont complétél'étude.

5 - Intervention(s) : la nature exacte de l'intervention, s'il y en a eu une, par exemple, un traitement (ou une mesure pédagogique).

6 - M esure des principaux résultats.

7 - Résultats: résultats principaux.

8 - Conclusions : conclusions principales, y compris les applications cliniques (ou pédagogiques).

Le groupe de travail a publié ses recommandations dans les Annals of I nternal M edicine et son rédacteur en chef, $E d w a r d ~ H$ uth, a créé le terme "résumé structuré» ${ }^{4}$. D epuis, la plupart des revues scientifiques ont emboîté le pas, en France tout autant que dans les pays de langue anglaise ${ }^{5}$, en utilisant le modèle de façon intégrale ou selon certaines variantes tout en conservant l'ensemble ou la plupart du contenu de chacune des rubriques originales. Le résumé structuré convient aussi bien au domaine de la pédagogie médicale qu'à celui de la médecine clinique ou fondamentale, et des revues comme M edical Education, Teaching and Learning in $M$ edicine et maintenant Pédagogie médicale n'ont pas hésité à l'adopter.

Le résumé structuré offre trois avantages principaux ${ }^{1}$ : 1 - aider les lecteurs à sélectionner plus rapidement des articles d'intérêt ;

2 - faciliter les recherches bibliographiques informatisées:

3 - faciliter la révision par les pairs.

Le résumé structuré fournit rapidement aux lecteurs, et aux évaluateurs externes, une vue d'ensemble de I'étude ainsi que certaines informations essentielles.

L'exemple ci-contre représente une illustration du principe pour un article de recherche (traduit et adapté avec la permission de M edical Education ${ }^{6}$ ).

$\mathrm{N}$ otez que deux rubriques ont été omises par le rédacteur en chef, soit «Interventions » (non pertinente ici) et « $M$ esure des résultats principaux » (incluse à même les Résultats). N otez aussi que le taux de réponse a été mis dans les Résultats plutôt que dans la section Participants. D e telles variantes sont fréquentes, toutefois la structure générale du résumé demeure intacte.

\footnotetext{
*Department of Medical Education - College of Medicine - University of Illinois at Chicago - Chicago, Illinois - Etats-Unis

**Département de médecine - Faculté de médecine - Université de Montréal - Montréal, Québec - Canada Correspondance : Georges Bordage - Department of Medical Education - College of Medicine - University of Illinois at Chicago - 808 South Wood (MC/591) - Chicago, III. 60612-7309 - Etats-Unis - Tél. : (312) 996-7349 - Télécopieur : (312) 413-2048 - Courriel : bordage@uic.edu
} 
La notion de résuméstructuré a aussi été mise de l'avant pour les articles de synthèse, en six points ${ }^{3,7}$ :

1- But : objectif principal de la revue de la littérature.

2- Sources des données.

3- Sélection des études : nombre d'études sélectionnées et critères de sélection.
4- Extraction des données : critères d'extraction des données.

5- Résultats de la synthèse des données : méthoded'analyse des données et résultats principaux.

6- Conclusions : conclusions principales, y compris les applications potentielles et les avenues de recherche futures.

\title{
Compétences et qualités de directeurs de programmes d'enseignement
}

\author{
G. Bordage, R. Foley \& S. Goldyn
}

But : Q uelles sont les compétences et qualités souhaitables chez un directeur de programme d'enseignement, de l'avis d'employeurs potentiels de futurs directeurs?

Plan de recherche: Enquête postale internationale menée à l'aide d'un questionnaire à question ouverte d'une page.

Contexte : D irecteurs de programme d'enseignement en science de la santé à l'échelle internationale.

Partici pants: $D$ oyens, directeurs de département et directeurs généraux en sciences de la santé.

Résultats : Un total de 139 participants ont rempli le questionnaire (taux de réponse de $22,3 \%$ ). Les réponses ont été remarquablement semblables d'une profession de la santé à une autre. Les neuf compétences le plus souvent mentionnées ont été : la communication orale, les relations interpersonnelles, la compétence clinique, l'élaboration d'objectifs pédagogiques, la planification pédagogique, la prise de décision et la résolution de problèmes, le travail en équipe, la communication écrite, et la planification budgétaire. Les trois qualités les plus prisées ont été : visionnaire, flexible et à l'esprit ouvert, et loyal.

Conclusions : La liste complète des compétences et qual ités peut servir à établir des profils d'embauche ou des listes de contrôle pour l'évaluation des directeurs de programme ou des programmes de formation pour directeurs. L'importance des qualités de chef de file est discutée ainsi que la nécessité d'une véritable formation des futurs directeurs de programme.

(N ombre de mots : 208) 
Voir l'exemple ci-dessous qui a été traduit et adapté avec la permission de Academic M edicine.

\title{
La transmission (tournée) du matin : objectifs et modalités au cours des trois dernières décennies.
}

\author{
Z. Amin, J. Guajardo, W. W isniewski, G. Bordage, A. Tekian \& L. Niederman
}

But : Le but de cette revue de la littérature concernant la transmission (tournée) du matin était d'en identifier les divers objectifs et modalités, de documenter sa valeur pédagogique et d'identifier certaines pistes futures de recherche.

Sourcesdes donnéeset sélection desétudes: Q uatre méthodes complémentaires ont été employées pour les articles publiés entre 1966 et décembre 1999 : recherches informatisées, recherches manuelles, vérification des références de certains articles et contacts personnels.

Extraction des données: Une démarche en trois temps a été employée : identification des problèmes clés, sélection des informations pertinentes et synthèse critique.

Résultats de la synthèe desdonnées: $Q$ uarante-huit articles ont étéidentifiés. Latransmission du matin peut avoir plusieurs objectifs : enseignement, évaluation des résidents et de la qualité des soins, détection et compte rendu d'événements indésirables, et socialisation. La fréquence, le moment et la durée de la transmission du matin sont semblables d'un programme à un autre, alors que la variété des participants, la direction des réunions, la sélection des cas, l'archivage et le suivi des malades diffèrent grandement. Plusieurs méthodes pédagogiques sont employées avec des résultats favorables.

Conclusions : Q uatre champs de recherche future ont été identifiés : la contribution unique dela transmission du matin au processus d'apprentissage, l'anal yse des niveaux positifs et négatifs de satisfaction par rapport à cette activité, son efficacité pédagogique et clinique et le besoin d'études regroupant plusieurs établissements. Enfin, le besoin d'étudier la transmission du matin en milieu ambulatoire est souligné.

(N ombre de mots : 239)

Academic M edecine 
II est bien établi que les résumés structurés contiennent un plus grand nombre d'informations que le résumé traditionnel non structurée, ${ }^{910}$. Cependant, certains chercheurs ont trouvé que plusieurs éléments importants sont souvent absents de ce type de résumé, même lorsqu'on les trouve dans le corps de l'article ${ }^{11}$. Pitkin et Branagan ${ }^{12}$ ont aussi démontré que malgré des directives précises visant à éviter trois erreurs courantes à savoir : (a) une contradiction entre le corps del'article et le résumé ; (b) un élément présent dans le résumé mais absent du corps del'article; (c) des conclusions non justifiées par les éléments présentés dans le résumé, ces erreurs persistent. Par conséquent, auteurs et évaluateurs externes doivent demeurer vigilants.

Enfin, le résumé structuré est un excellent outil de rédaction. Très vite, les auteurs peuvent entreprendre la rédaction du corps de l'article en complétant chacune des rubriques du résumé structuré à l'aide d'abord de mots clés. Ce premier jet donnera aux auteurs une vue d'ensemble du travail à accomplir et servira de point de repère tout au long du processus de rédaction, en facilitant l'agencement et l'articulation des différentes composantes de l'article ; ainsi, ce jet structuré sera une source immédiate de satisfaction qui facilitera la mise en route du processus de rédaction, celui-ci pouvant être pénible au point de départ.

En somme, le résumé structuré est un excellent outil de lecture, d'évaluation et de rédaction. Tous y gagnent, auteurs, rédacteurs en chef, évaluateurs externes et lecteurs !

\section{Références}

1.Ad H oc Working Group for Critical Appraisal of the M edical Literature. A proposal for more informative abstracts of clinical articles. Annals of Internal M edicine 1987 ; 106 : 598-604.

2.Altman D G, Gardner MJ. M ore informative abs tracts (letter). Annals of Internal M edicine 1987 ; $107: 790-791$.

3.H aynes RB, M ulrow CD, H uth EJ, Altman DG, Gardner $M J$. M ore informative abstracts revisited. Annals of Internal M edicine $1990 ; 113$ : 69-76.

4.H uth EJ. Structured abstracts for papers reporting clinical trials. Annals of Internal M edicine 1987 ; $106: 626-627$.

5. Revuz $\mathrm{J}$. Résumés structurés et résumés informatifs. Ann D ermatol Venereol $1993 ; 120: 279-280$.

6.Bordage G, Foley R, Goldyn S. Skills and attributes of directors of educational programmes. Medical Education $2000 ; 34$ : 206-210.

7.M ulrow CD, Thacker SB, Pugh JA. A proposal for more informative abstracts of review articles. Annals of Internal M edicine $1988 ; 108: 613-615$.

8.Zubair A, Guajardo J, Wisniewski W, Bordage G, Tekian A, N i ederman L. M orning report : focusand methods over the past three decades. Academic M edicine $2000 ; 75:$ S1-S5.

9.C omans $M$ L, $O$ verbeke $A$ J. T he structured summary : A tool for reader and author. N ed Tijdschr Geneeskd $1990 ; 134: 2338-43$.

10.Taddio A, Pain T, Fassos FF, Boon H, Ilersich $A L$, Einarson $T R . Q$ uality of nonstructured and structured abstracts of original research articles in the British M edical Journal, the Canadian M edical Association Journal and the Journal of the American M edical Association. Canadian M edical Association Journal $1994 ; 150: 1611-14$.

11.Froom P, Froom J. D eficiencies in structured medical abstracts. Journal of Clinical Epidemiology $1993 ; 46: 591-94$.

12.Pitkin RM , Branagan M A. Can the accuracy of abstracts be improved by providing specific instructions? A randomized controlled trial. Journal of the American M edical Association 1998 ; 280 : 267269. 